Check for updates

Cite this: RSC Adv., 2018, 8, 34895

\title{
Surface analysis of curved polymeric plates irradiated with proton and ion beams $\uparrow$
}

\author{
Young Seok Song, ${ }^{a}$ Chul Kang, ${ }^{a}$ Jiwon Jeong, ${ }^{a}$ Minji Kim ${ }^{\mathrm{b}}$ and Eunju Lim (D) *b
}

Received 7th August 2018

Accepted 19th September 2018

DOI: $10.1039 / \mathrm{c} 8 \mathrm{ra06636a}$

rsc.li/rsc-advances

\begin{abstract}
We explored surface characteristics of polycarbonate substrates which were subjected to proton and nitrogen ion beams. Curved plates designed for a plastic window of a cell phone were fabricated using injection molding, and the process was simulated numerically. Different energy densities of proton and nitrogen ion beams were applied in this study. The physical and chemical properties of the samples were evaluated using UV-Vis spectroscopy, FT-IR, differential scanning calorimetry (DSC), nanoindentation, residual stress, and electrical analyses. Results revealed that the irradiation of proton and nitrogen ion beams led to changes in the chemical structures and energy levels of the polymer molecules. Furthermore, physical properties, such as hardness, glass transition temperature, transmittance, and electrical conductivity, were remarkably varied.
\end{abstract}

\section{Introduction}

Ion implantation is a powerful method for modifying materials by bombarding them with ion beams such as protons, argon, nitrogen, and metals. Since this can control energy power, fluence, and profile of irradiated ion/dopants, many relevant studies are being carried out these days. However, understanding the microstructural changes induced by ion beam implantation in materials still needs to be developed for advanced manufacturing processes for high performance materials. ${ }^{\mathbf{1 - 4}}$

Polymeric materials are widely employed in various applications due to their fascinating physical and chemical features such as high specific modulus and strength, high transparency, high chemical resistance, flexibility, and lightweight properties. Furthermore, they can be produced in time and cost effective ways using efficient polymer processing methods such as injection molding and extrusion. ${ }^{5-8}$ These days, many attempts are being made to replace metal and/or ceramic materials with plastics in a wide range of applications such as in electronics, automobiles, optics, and aeronautics. ${ }^{\mathbf{9} 10}$ Among plastics, polycarbonate (PC) with excellent transparency and high impact strength has been extensively used in a wide range of such applications including microelectronics, medical devices, biosensors, and photonics. However, these polymeric materials have several problems to resolve, e.g., relatively low durability and anti-aging ability. On the other hand, aromatic polymers such as PC show relatively high resistance to ion beams, X-rays, and gamma rays. ${ }^{\mathbf{1 , 1 1 , 1 2}}$

${ }^{a}$ Department of Fiber System Engineering, Dankook University, Yongin 448-701, Korea ${ }^{b}$ Department of Science Education/Creative Convergence Manufacturing Engineering, Dankook University, Yongin 448-701, Korea. E-mail: elim@dankook.ac.kr; Tel: +8231-8005-3217

$\dagger$ Electronic supplementary information (ESI) available. See DOI: 10.1039/c8ra06636a
There is a growing interest for enhancing the surface properties in polymer science and engineering. In particular, ion implantation is capable of engineering and improving the surface properties of polymers. During ion irradiation, various phenomena in a polymer may take place, such as macromolecular chain scission, cross-linking, free radical formation, carbonization, oxidation, and so on. In other words, polymer chains might be destructed and re-arranged by deposited energy through the ion bombardment, leading to a change in polymeric characteristics such as phase, molecular structure, crystallinity, and molecular weight. ${ }^{13}$ Generally, chain scission of polymer molecules decreases molecular weight and crosslinkings are likely to increase the weight. ${ }^{14-17}$ E. H. Lee explained the cross-linking mechanism induced by the irradiation of high energy ion beams and reported enhancement in hardness of the samples. ${ }^{18}$ When an ion beam is irradiated onto a polymer, the beam energy diffuses into the substrate. In the case of ion implantation, two kinds of stopping powers (i.e., energy loss) can be taken into account. First, electronic stopping power can be explained by interactions between the incident ions and electrons of the polymer. ${ }^{\mathbf{1 9 , 2 0}}$ Second, nuclear stopping power results from Coulomb interactions between the nuclei of material and the incident particles. ${ }^{21-23}$ The ion irradiation generates irreversible changes of polymer configuration and forms reactive elements such as radicals.

In this study, we analyzed the surface properties of curved polymeric parts irradiated with proton and nitrogen ion beams. The curved parts were designed for application as a plastic window for a cell phone and prepared using injection molding, and various fluences were considered in the experiments. Physiochemical properties of PCs treated with an ion beam were evaluated and fabrication processes of the specimens were simulated numerically. Unlike literature regarding ion beam irradiated polymers, this report sheds light on the change in mechanical 
properties of plastic parts such as residual stress and hardness along with the fabrication process of the specimens.

\section{Experimental}

\section{Materials and sample preparation}

PC was supplied by Lotte Chemical Co (Korea) (PC-1150). It encompasses pellets with a diameter of around $1 \mathrm{~mm}$. Prior to the injection molding process, the pellets were completely dried. The drying conditions were $100{ }^{\circ} \mathrm{C}$ for 4 hours. The moisture content was less than $0.2 \%$. The polymer melt temperature was $300^{\circ} \mathrm{C}$, and the mold temperature was $80^{\circ} \mathrm{C}$. The mold was pre-warmed for 3 hours to secure enough time for thermal equilibrium.

\section{Beam implantation}

The injection molded PC substrate had a thickness of $0.6 \mathrm{~mm}$ and a curved shape as shown in Fig. 1. Fig. S1† shows the chemical structure of PC. Proton and nitrogen ion beams were irradiated towards the prepared samples with an energy 10.5 $\mathrm{MeV}$ and proton doses of $1 \times 10^{11}$ to $1 \times 10^{14}$ protons per $\mathrm{cm}^{-2}$ and with an energy of $20 \mathrm{keV}$ and ion doses of $1 \times 10^{14}$ to $1 \times$ $10^{17}$ ions per $\mathrm{cm}^{-2}$, respectively (Fig. S2 $\dagger$ ). Sample size for the beam irradiation was $2 \times 2 \mathrm{~cm}^{2}$. The ion implanter was comprised of a Duo-Pigatron ion source, and accelerating tube, and a vacuum chamber. The applied vacuum level was $1 \times 10^{-5}$ torr during idle condition and $5 \times 10^{-5}$ torr during irradiation.

\section{Measurements}

Transmittance of the samples was evaluated at wavelengths in the range of $250 \mathrm{~nm}$ to $1000 \mathrm{~nm}$ using a UV-Visible spectrometer (Lambda 1050, PerkinElmer). The Fourier transform infrared (FT-IR) spectrum of the samples was analyzed using a Nicolet 6700, Thermo Scientific FT-IR spectrometer. A KBr-pellet method was used, and the scan range was $4000-400 \mathrm{~cm}^{-1}$. The thermal characteristics of specimens were investigated using differential scanning calorimetry (DSC, Q2000 system, TA Instruments). The specimens for the DSC experiment were scanned between $25{ }^{\circ} \mathrm{C}$ and $300{ }^{\circ} \mathrm{C} \pm 10{ }^{\circ} \mathrm{C} \mathrm{min}{ }^{-1}$.

Nanoindentation experiments were performed using a high temperature ultra-nanoindentation tester (UltraNano, CSM). A Berkovich tip was used, and a quasi-static load function with a peak force of $500 \mu \mathrm{N}$ was applied. At least 5 tests were repeated and averaged. Electrical conductivity measurements were measured using a semiconductor parameter analyzer (HP 4155A) and voltage was applied from the positive to negative directions. In order to evaluate the deposited energy effect by proton and nitrogen ion beam irradiations, the samples were placed on a hot plate at $200{ }^{\circ} \mathrm{C}$ for 1 hour, and then their absorption spectra were scanned.

Residual stress of the samples was measured using a holedrilling method. In order to obtain a representative profile for residual stress in the thickness direction, the test was carried out at the centers of the samples. A strain gauge rosette was fixed on the surface of injection molded parts using cyanoacrylate adhesive. A drill was positioned at the center of the rosette using an optical microscope. Holes were drilled in depth (a)

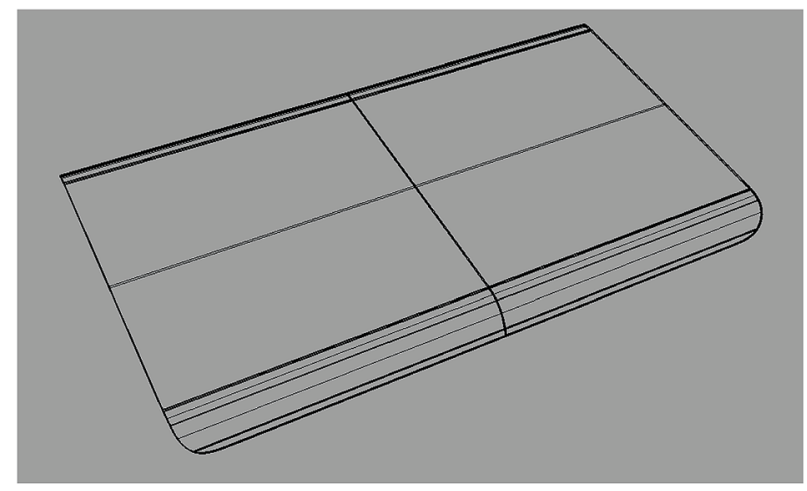

(b)

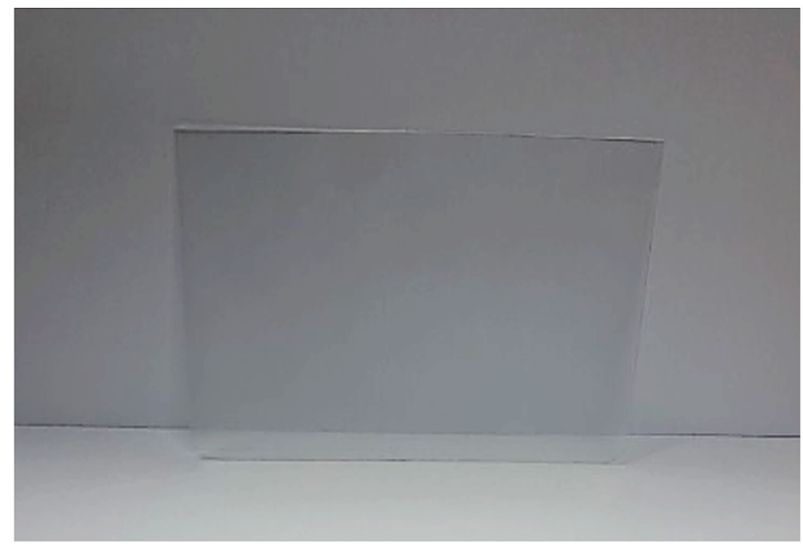

Fig. 1 (a) Design of a curved polymeric part and (b) photograph of the part fabricated in this study. The window size was 6 inch.

increments of $0.1 \mathrm{~mm}$ with a radius of $0.94 \mathrm{~mm}$. At each depth, about 3 min was given to stabilize the samples prior to the measurements. The residual stresses in a specimen was then calculated at each hole depth using the results of the strain gauge. The residual stresses are expressed as below: ${ }^{24}$

$$
\sigma=\frac{\varepsilon_{1}+\varepsilon_{2}}{4 A} \pm \frac{\sqrt{2}}{4 B} \sqrt{\left(\varepsilon_{1}+\varepsilon_{2}\right)^{2}+\left(\varepsilon_{2}-\varepsilon_{3}\right)^{2}}
$$

where $A=-a(1-\nu) / 2 E, B=-b / 2 E, \sigma$ is the maximum principal stress at the hole location. Here, $\varepsilon_{1}, \varepsilon_{2}$, and $\varepsilon_{3}$ are the relieved strains measured from radially orientated strain gauges. For more details, $\varepsilon_{1}$ and $\varepsilon_{3}$ are the values along the two principal axes perpendicular to each other, and $\varepsilon_{2}$ is the value at $45^{\circ}$ to the two principal axes. $E$ and $\nu$ are the modulus and Poisson's ratio of the injection molded part, respectively, and $a$ and $b$ are dimensionless calibration coefficients.

\section{Numerical simulation}

The manufacturing process of the curved polymeric plates was simulated numerically. For it, governing equations are as below: ${ }^{25}$

$$
\frac{\mathrm{d} \rho}{\mathrm{d} t}+\rho(\nabla \cdot \tilde{v})=0
$$



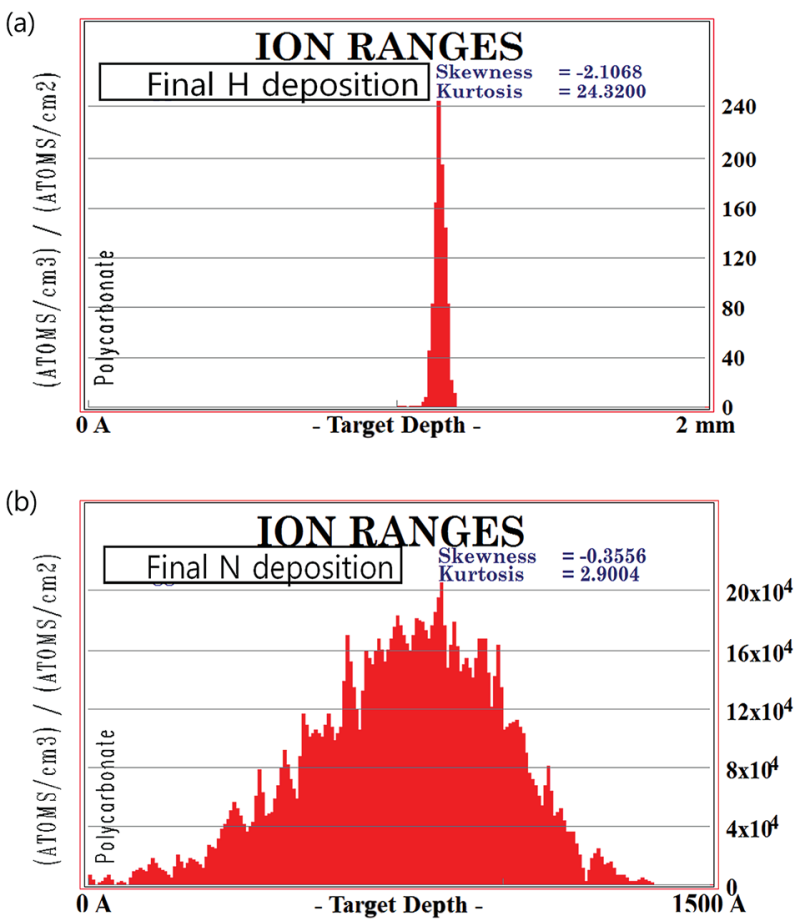

Fig. 2 Results of the SRIM simulation: penetration depth profile of (a) proton and (b) nitrogen ion beams.
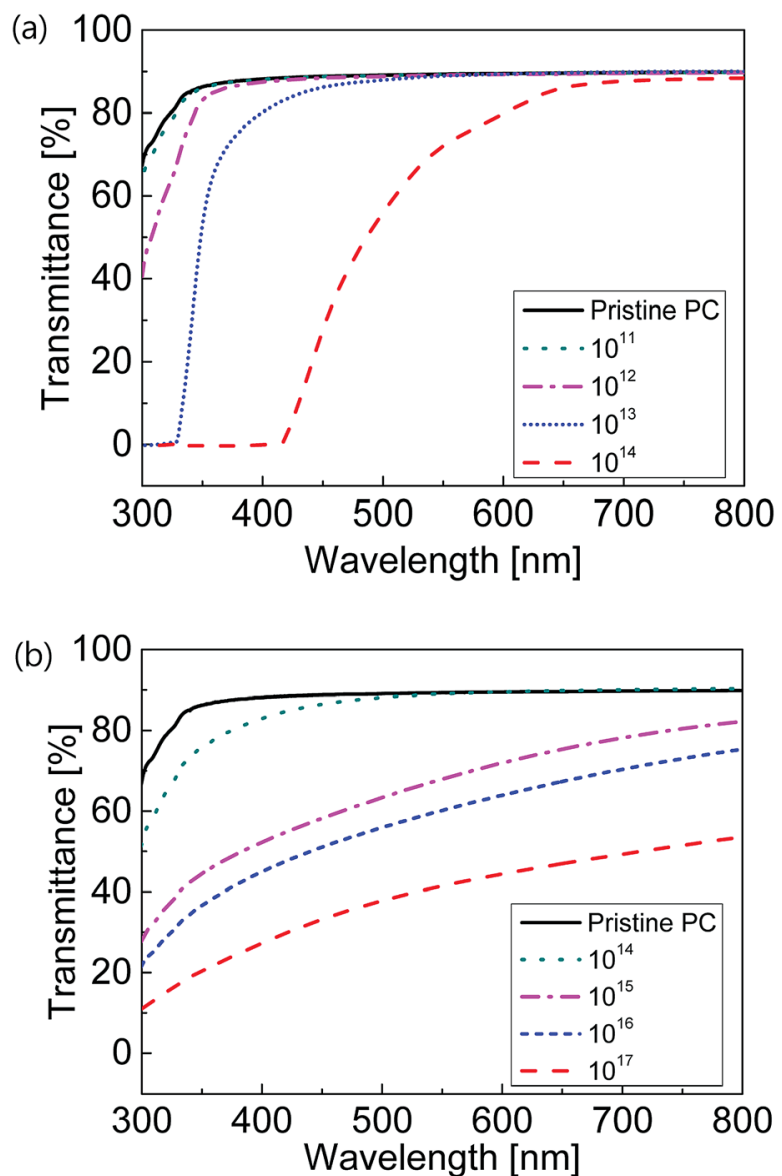

Fig. 3 UV-Vis transmittance spectra of the samples irradiated with (a) proton and (b) nitrogen ion beams.
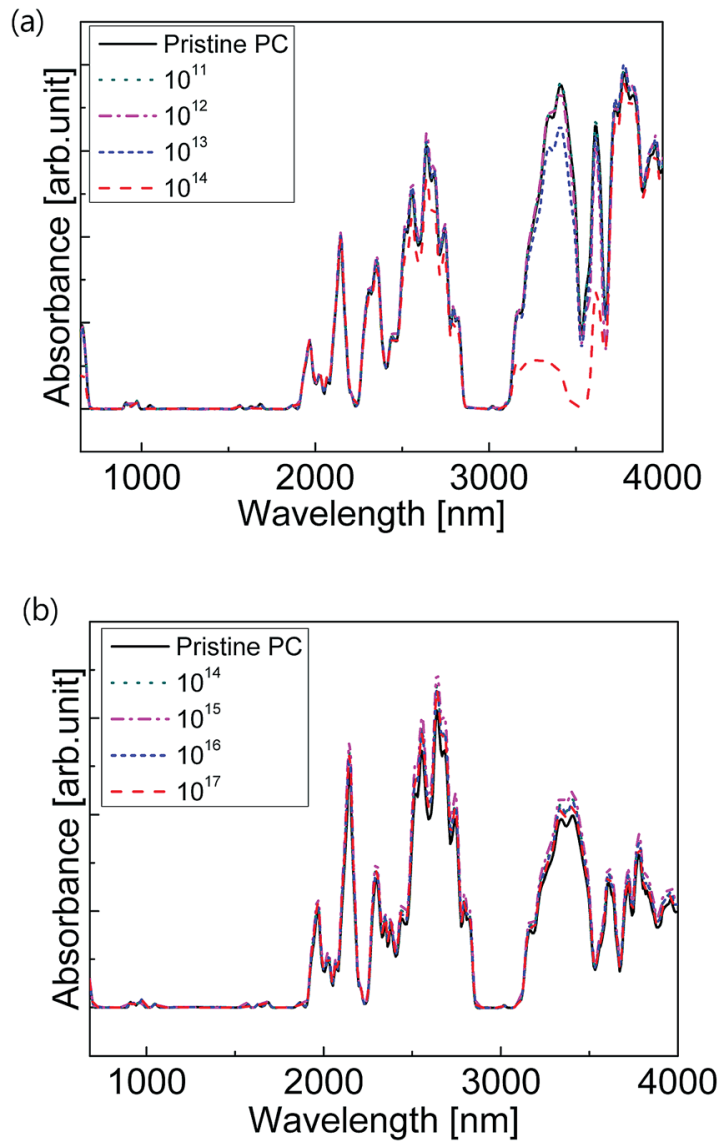

Fig. 4 FT-IR spectra of the samples irradiated with (a) proton and (b) nitrogen ion beams.

$$
\begin{gathered}
\rho \frac{\mathrm{d} \tilde{v}}{\mathrm{~d} t}=-\nabla P+\nabla \cdot \tilde{\tau}+\rho \tilde{g} \\
\rho C_{\mathrm{p}} \frac{\mathrm{d} T}{\mathrm{~d} t}=\beta T \frac{\mathrm{d} P}{\mathrm{~d} t}+\eta \dot{\gamma}^{2}+\nabla \cdot \tilde{q}
\end{gathered}
$$

where $\rho$ is the density, $\tilde{v}$ is the velocity vector, $P$ is the pressure, $\tilde{\tau}$ is the viscous stress tensor, $\tilde{g}$ is the gravity/body-force vector, $C_{\mathrm{p}}$ is the specific heat at constant pressure, $\beta$ is the coefficient of thermal expansion, and $\tilde{q}$ is the heat flux. Since the polymer melt was regarded as a non-Newtonian viscous fluid in this study, the viscosity, $\eta$ can be expressed using the following modified Cross model.

$$
\eta(T, \dot{\gamma}, P)=\frac{\eta_{0}(T, P)}{1+\left(\eta_{0}(T, P) \frac{\dot{\gamma}}{\tau^{*}}\right)^{1-n}}
$$

where $\eta_{0}$ is the zero shear rate viscosity, and $\tau^{*}$ is the shear stress at the transition between the Newtonian and the Power law behaviors, and $n$ is the Power law index.

\section{Results and discussion}

Fig. 2 shows the penetration profiles of proton and $\mathrm{N}_{2}$ ion beams calculated with the Stopping and Range of Ions in Matter (SRIM) program. To predict ion implantation effects in 
polymers, a Monte Carlo simulation was carried out. ${ }^{1}$ The density of the PC was $1.21 \mathrm{~g} \mathrm{~cm}^{-3}$. The penetration depths of proton and $\mathrm{N}_{2}$ ion beams into the PC substrate were $1.13 \mathrm{~mm}$ and $72.5 \mathrm{~nm}$, respectively. When the beams enter inside the matrix, they collide with carbon and hydrogen atoms and recoil. For a statistical understanding on stopped positions of the implanted ions, the density of ions is demonstrated as a function of target depth. Since the thickness of the sample was 0.66 $\mathrm{mm}$, the proton beams could permeate the sample but the $\mathrm{N}_{2}$ ion beams were stopped around surface of the sample.

Fig. 3 presents the UV-Vis transmittance spectra of the ion beam irradiated samples. As the ion doses increased, the spectra decreased with respect to the wavelength. This decrement is related to the color change to brownish yellow in the samples, which results from the formation of color sensitive conjugated bonds with loosely bound $\pi$ electrons and an increase of ion absorption. The samples implanted with proton ion beams were found to have larger transmittance and frequency dependence than those treated with nitrogen ion beams. In particular, transmittance of the proton ion specimen with a fluence of $1 \times 10^{14}$ dramatically changed according to the wavelength. The transmittance reduction can be understood based on the changes in chemical structures of the samples. As a result of the ion irradiation, chain scission occurs in the
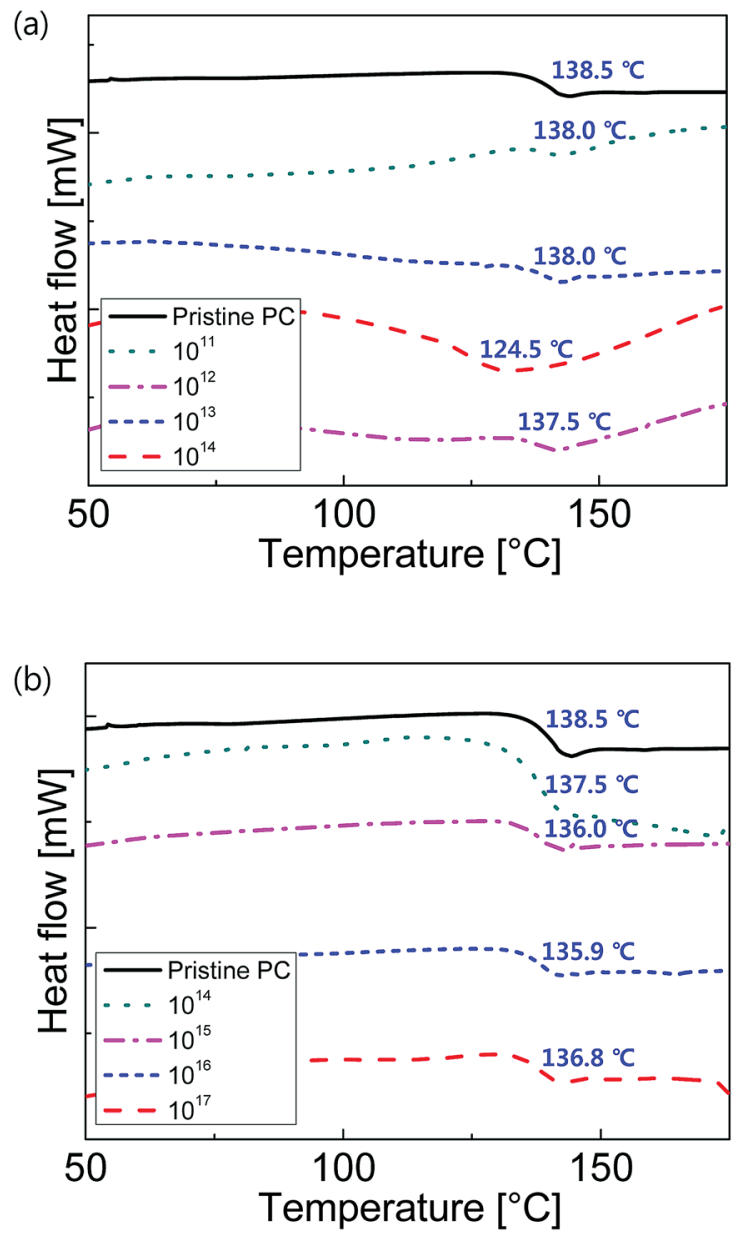

Fig. 5 DSC thermograms of the samples treated with (a) proton and (b) nitrogen ion beams. polymer and free radicals are generated. Thereafter, new chemical bonds by cross-linking or defects can be formed.

FT-IR spectra of the samples are shown in Fig. 4. Overall, transmittance decreased with increasing ion fluence. This reduction is because of the cleavage of carbon bonds, dehydration, and formation of new linkages. Similar to the results of UVVis spectroscopy, the proton ion beam led to a larger decrease in the FT-IR absorbance than the $\mathrm{N}_{2}$ ion beam. As the ion density of protons increased up to $10^{14}$ protons per $\mathrm{cm}^{-2}$, the absorbance of the proton ion implanted PC remarkably decreased in the wavelength range of 3000 to $3700 \mathrm{~nm}$. This range contains the characteristic peaks of aromatic, $\mathrm{C}-\mathrm{H}$, and $\mathrm{O}-\mathrm{H}$. Such a decline in spectrum intensity implies a change of chemical structure and the resulting compositional changes. It is assumed that the chemical change induces remarkable changes in physical properties of the parts prepared using injection molding in this study. As aforementioned, optical properties such as transmittance are highly dependent on the chemical structure and configuration of molecules. Proton beam irradiation resulted in chain scission, thus decreasing the transmittance of a specimen as demonstrated in Fig. 3(a). In contrast, samples implanted with $\mathrm{N}_{2}$ ion beams showed a slight change in absorbance when the fluence increased even up to $10^{17}$ ions per $\mathrm{cm}^{2}$.

Fig. 5 shows DSC thermograms of the ion beam irradiated specimens. It turned out that the glass transition temperatures gradually decreased with an increase in the fluences of proton and nitrogen ions.

(a)

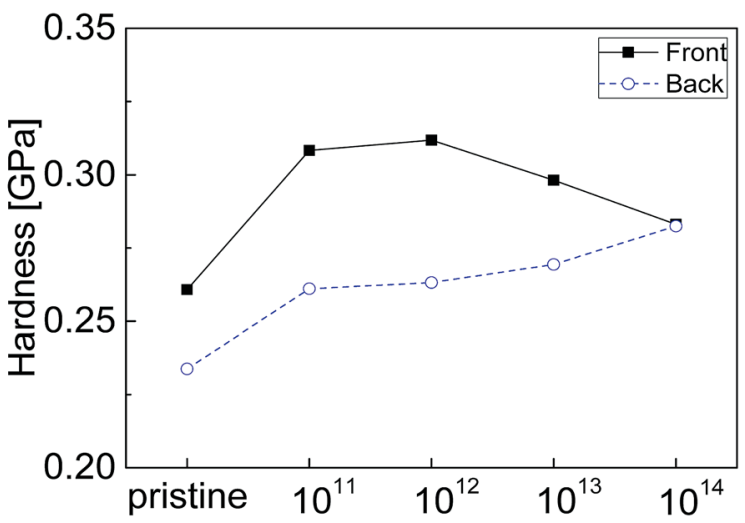

(b)

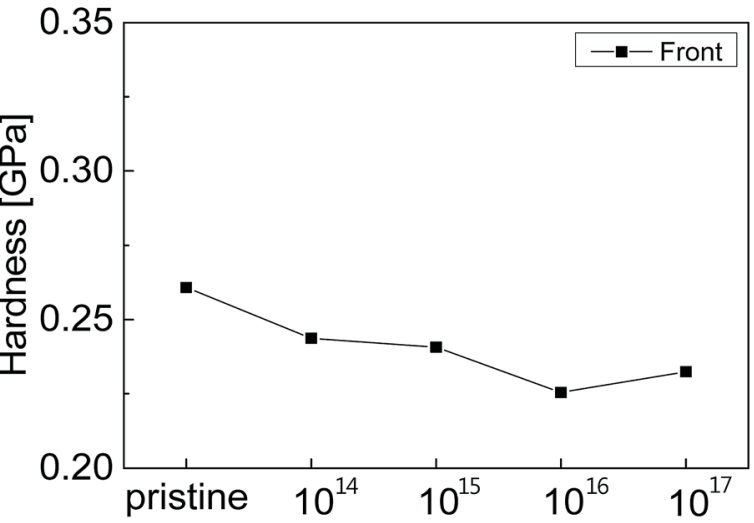

Fig. 6 Hardness of PC irradiated with (a) proton and (b) nitrogen ion beams. 
Such decrease was more obvious in the case of the proton ion beam than in that of the nitrogen ion beam. Proton and $\mathrm{N}_{2}$ ion beam irradiations change the microstructures of polymers, such as crystal structure. Also, ion beam irradiation causes

(a)

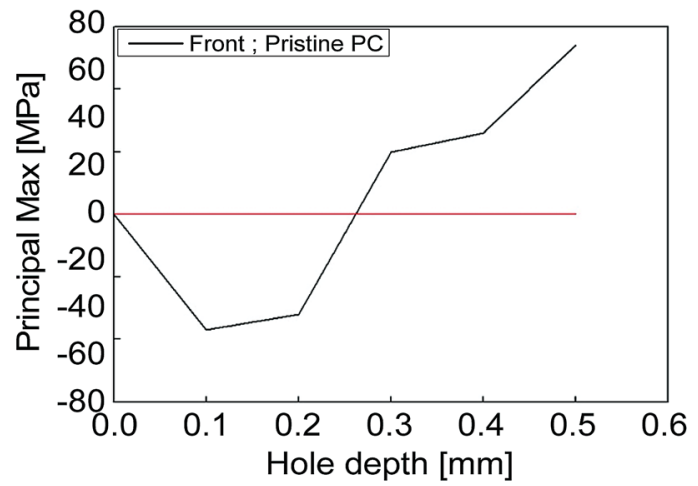

(b)

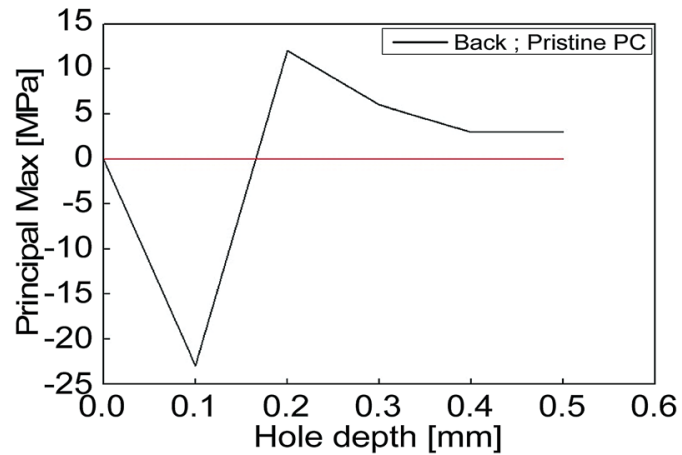

(c)

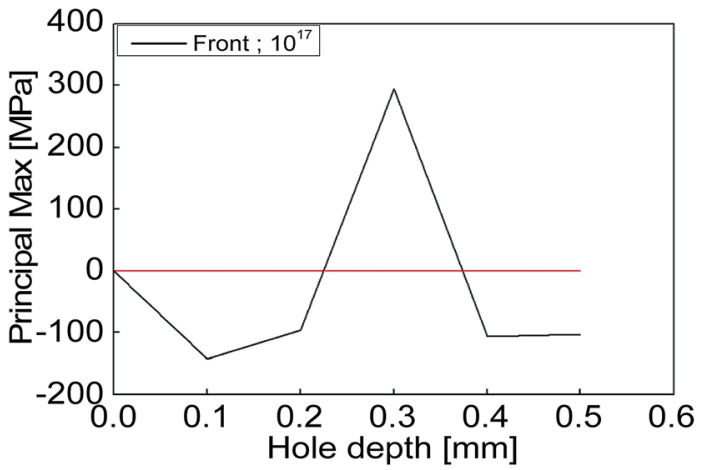

(d)

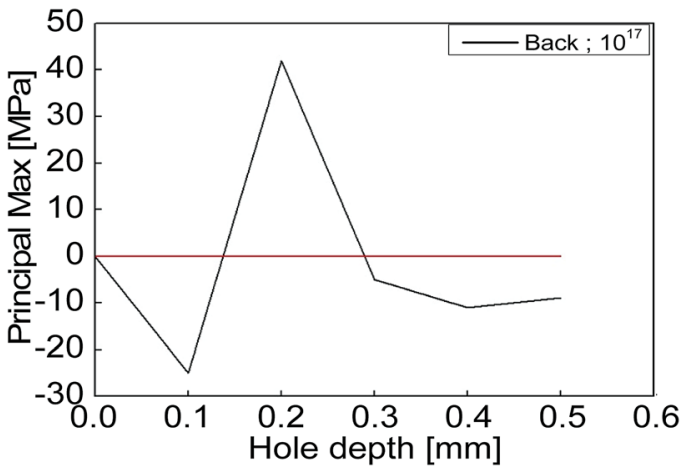

Fig. 7 Profiles of residual stress as a function of depth: ( $a$ and b) front and back sides for pristine PC and (c and d) front and back sides for nitrogen ion irradiated PC, respectively. chain scission of polymers, thereby increasing the mobility of molecules. For proton irradiated samples, the lowest glass transition temperature was observed at $10^{14}$ ions per $\mathrm{cm}^{2}$. In the case of the nitrogen ion beam, a decrease in the glass transition temperature is, however, not significant.

Fig. 6 demonstrates the hardness results of PCs implanted with ion beams. Since the polymeric part prepared in this study had a curved shape, it experienced asymmetric cooling during injection molding. Therefore, the magnitude of residual stress in the front side was larger than that in the back side. In this sense, the front side had higher hardness than the back side. As the proton ion beam irradiation was increased, the hardness of the back side decreased. On the contrary, the hardness of the front side showed more complex behavior, e.g., a maximal value. Note that hardness relies upon not only the chemical structure of a polymer but also the state of residual stress. Hence, mechanical properties need to be determined considering chemical changes including chain scission and crosslinking by the ion beam implantation and residual stress determined by the geometry and manufacturing conditions. Fig. 6(b) shows the decrement in the hardness with respect to the density of nitrogen ions.

Residual stresses resulting from the samples are displayed in Fig. 7. While a symmetric wall temperature on a mold was applied during injection molding, the part was not solidified

(a)

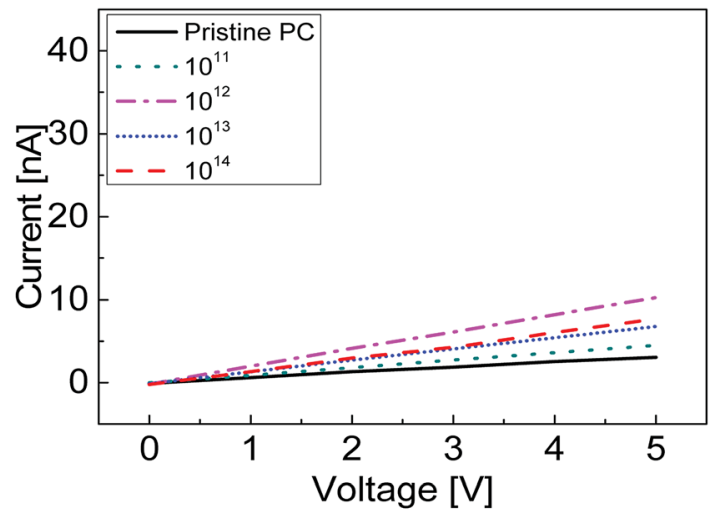

(b)

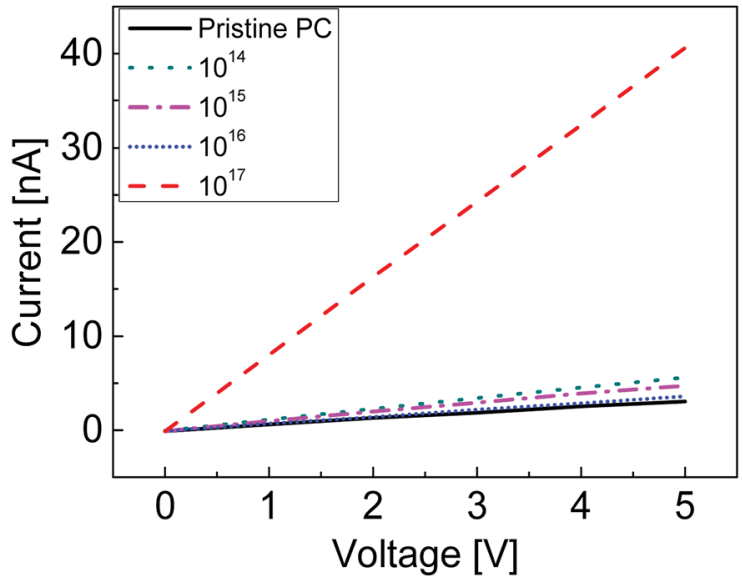

Fig. 8 Electrical conductivities of PC irradiated with (a) proton ion beam and (b) nitrogen ion beam. 
(a)

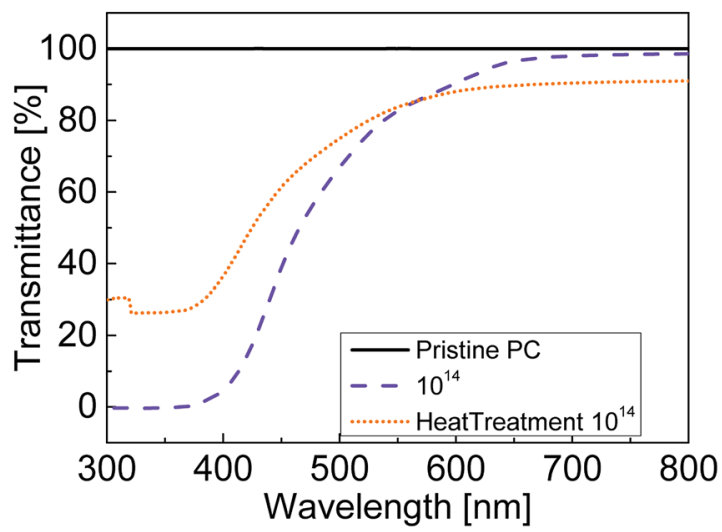

(b)

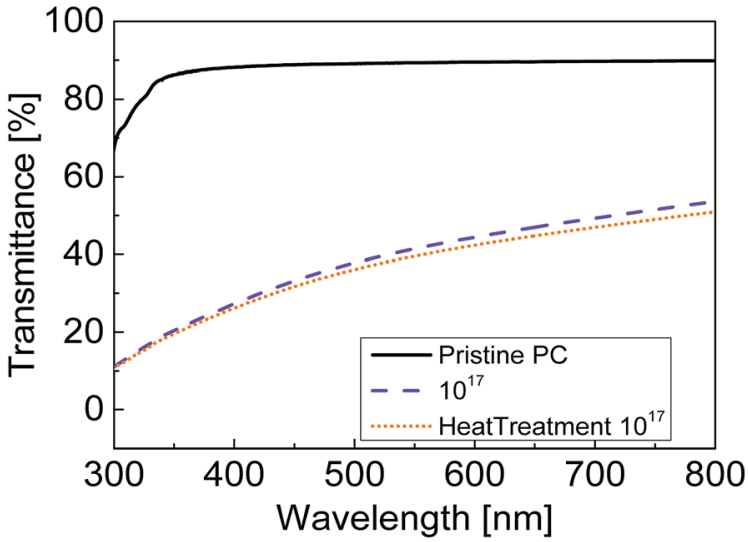

Fig. 9 UV-Vis transmittance spectra after heat treatment for (a) proton and (b) nitrogen ion beam irradiated samples.

uniformly along the thickness direction due to the curved geometry of the injection molded part. From Fig. 7(a) and (b), it was found that the residual stress in the front side was larger than that in the back side. This was due to relatively slow cooling of the back side, compared with the front side. Once a nitrogen beam was irradiated onto the sample, the states of residual stress in the front and back sides were changed. Hardness measured from the nanoindentation test is mostly determined by surface properties. Therefore, it is not easy to fully understand the hardness behavior of the samples since residual stress at the surface was not measured in this study.

Fig. 8 illustrates electrical conductivities of the PCs irradiated with proton and nitrogen ion beams. In most cases, electrical conductivities were found to increase by increasing the dose. In particular, when the $\mathrm{N}_{2}$ ion beam with a fluence of $10^{17}$ ions per $\mathrm{cm}^{-2}$ was applied, the electrical conductivity of the sample increased by almost 9 times more than the pristine PC. As stated above, a proton beam could pass through the entire specimen, but a nitrogen beam halts around the PC's surface. Therefore, most of the nitrogen ions remained on the surface, which could lead to such increase in the electrical conductivity. Furthermore, since the proton and nitrogen ion beams entailed a change of molecular structure (e.g., carbonization) as shown in Fig. 5, the corresponding conductivity could be enhanced. ${ }^{16}$
After heat treatment, UV-Vis transmittances of the samples irradiated by the proton and nitrogen ion beams are presented in Fig. 9. In the case of the sample implanted with the proton ion beam, the transmittance was fairly recovered especially in the regime of short wavelengths. This indicated that the proton beam

(a)

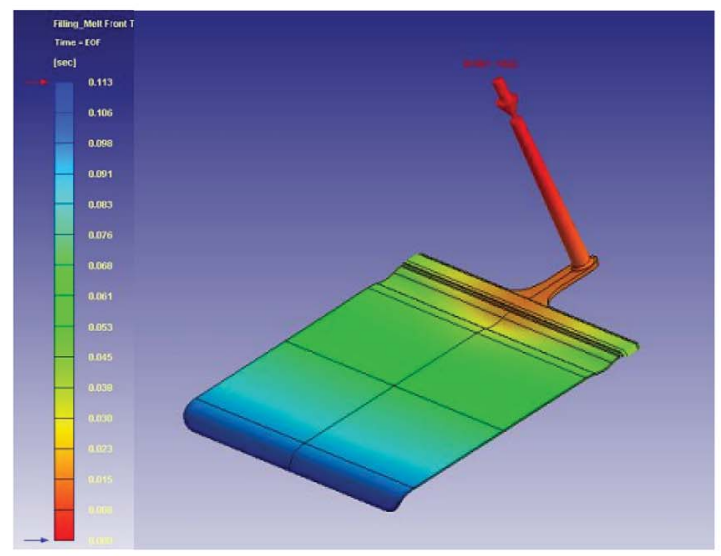

(b)

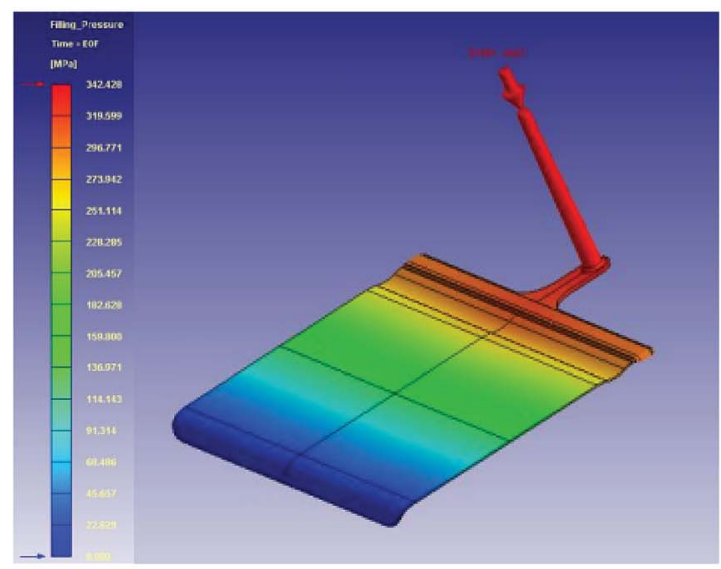

(c)

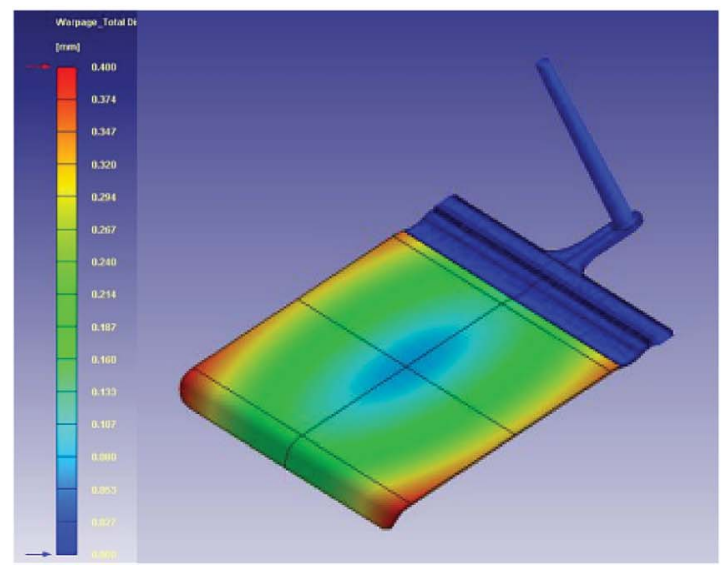

Fig. 10 Numerical results: (a) distribution of flow front time, (b) pressure field, and (c) total displacement in warpage. 
(a)
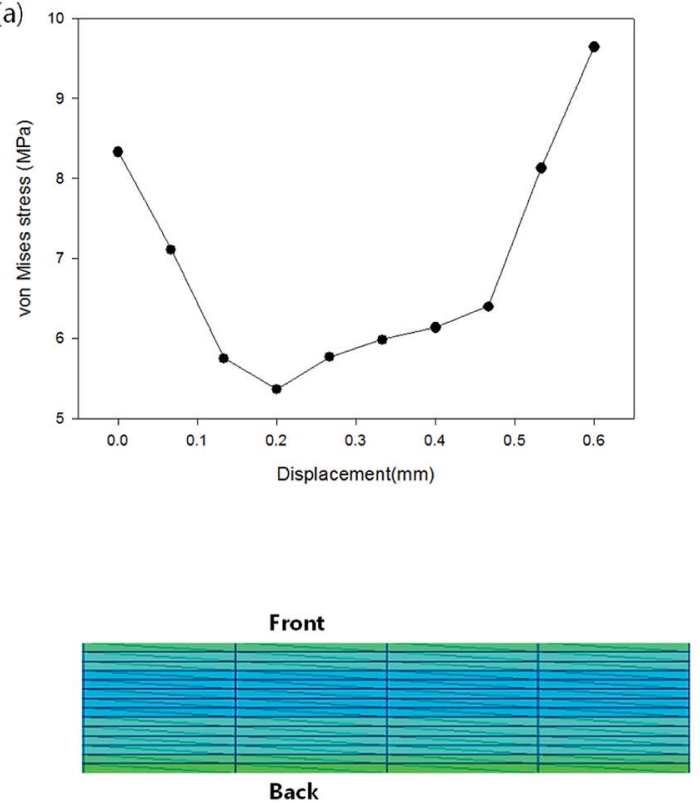

(b)

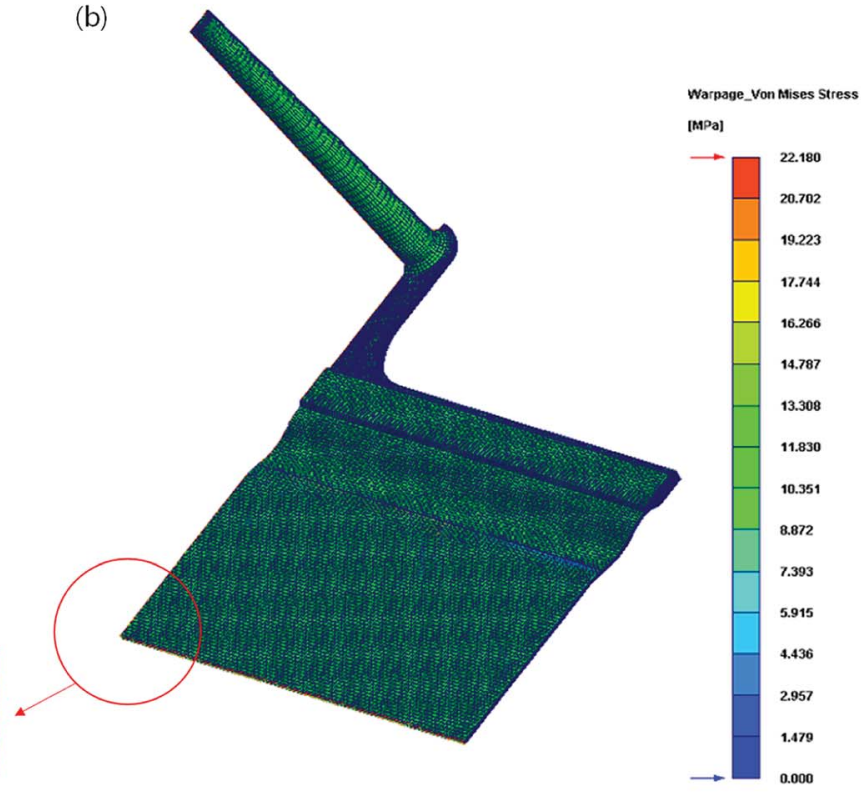

Fig. 11 Numerical results: (a) profile of von Mises residual stress along the sample thickness and (b) contour of the residual stress in the sample.

changed the energy state of the PC sample as well as its chemical structure. However, transmittance of the nitrogen ion beam treated sample was not changed after heat treatment. The decrease in transmittance induced by the nitrogen ion beam was associated with the denaturation of chemical structure rather than the energy state of material. Fig. 10 presents the numerical results of the injection molded parts. In this simulation, a single cavity was considered. The distributions of flow front time and pressure in the curved mold cavity are demonstrated in Fig. 10(a) and (b). Since the polymeric part had a curved shape, more complex patterns of the total displacement and residual stress were developed than with the flat geometry case. Displacement around the edges of the parts were found to be relatively large. Fig. 11 demonstrates numerical results of the residual stress developed in the sample. Please note that the residual stress calculated numerically was the von Mises stress but not principle stresses. The numerically obtained stress was found to have a similar profile shape to the experimental results. To better visualize the residual stress distribution at the center of the entire simulation domain, the domain was partitioned along flow and transverse directions. Fig. 11(b) shows the contour of the residual stress at the center and the enlarged distribution along the thickness after partitioning. Note that all the samples for the experiments were prepared from the center of the injection molded parts. Since the molded plates were curved, the mechanical properties of the front and back sides for the specimens were significantly different, such as hardness and residual stress. This implied that the curved shape led to a change in the microstructure of the samples. However, in other experiments such as FT-IR, DSC, UV-Vis, and electric tests the resulting change was not detectable. Overall, this study is expected to provide a meaningful fundamental understanding regarding the effects of ion beam irradiation on the physical properties of injection molded parts.

\section{Conclusions}

In this study, we investigated the surface properties of injection molded parts after implanting proton and nitrogen ions. For electronic applications, such as a cell phone and tablets, a curved plastic window was designed and manufactured via injection molding. The manufacturing process was also simulated numerically. The penetration depths of the proton and nitrogen ion beams were predicted. Various properties of the samples were analyzed through UV-Vis spectroscopy, FT-IR, DSC, and electrical conductivity measurements. The findings showed that implantation of proton and nitrogen ion beams changed not only the chemical structures of the samples but also the energy levels of polymer molecules. Consequently, such changes could yield significant variations in mechanical, thermal, and optical properties. We anticipate that this study could open a way for developing a polymeric part with outstandingly good physical properties at the interface between polymer engineering and quantum physics.

\section{Conflicts of interest}

There are no conflicts to declare.

\section{Acknowledgements}

The present research was conducted by the research fund of Dankook University in 2017. Also, this work was supported by the Basic Research Program (2015R1D1A1A02062233) of the National Research Foundation (NRF) funded by the Ministry of Education, Science and Technology, Korea. This work was supported by GRRC program of Gyeonggi Province (GRRC Dankook2016-B03). In addition, this work was supported by the 
National Research Foundation of Korea (NRF) grant funded by the Korea government (MSIT) (No. NRF-2018R1A5A1024127).

\section{References}

1 A. M. A. Reheem, M. I. A. A. Maksoud and A. H. Ashour, Radiat. Phys. Chem., 2016, 125, 171-175.

2 R. M. Radwan, A. M. Abdul-Kader and A. El-Hag Ali, Nucl. Instrum. Methods Phys. Res., Sect. B, 2008, 266, 3588-3594.

3 M. I. Chipară, V. V. Grecu, P. V. Notingher, J. Reyes Romero and M. D. Chipară, Nucl. Instrum. Methods Phys. Res., Sect. B, 1994, 88, 418-422.

4 E. Occhiello and F. Garbassi, High energy density technologies in material science, Dordrecht, 1990.

5 T. Sharma, S. Mahendia, S. Aggarwal, S. Kumar and D. Kanjilal, Opt. Mater., 2011, 33, 1741-1744.

6 G. Marletta, S. Pignataro and C. Oliveri, Nucl. Instrum. Methods Phys. Res., Sect. B, 1989, 39, 792-795.

7 S. Iqbal, M. S. Rafique, N. Iqbal, S. Bashir, S. Arif and R. Ahmad, Prog. Org. Coat., 2017, 111, 202-209.

8 P. Bertrand, P. Lambert and Y. Travaly, Nucl. Instrum. Methods Phys. Res., Sect. B, 1997, 131, 71-78.

9 P. Peinado, S. Sangiao and J. M. De Teresa, ACS Nano, 2015, 9, 6139-6146.

10 Y. Hama, K. Hamanaka, H. Matsumoto, T. Takano, H. Kudoh, M. Sugimoto and T. Seguchi, Radiat. Phys. Chem., 1996, 48, 549-554.

11 F. Dehaye, E. Balanzat, E. Ferain and R. Legras, Nucl. Instrum. Methods Phys. Res., Sect. B, 2003, 209, 103-112.
12 M. Mujahid, P. Singh, D. S. Srivastava, S. Gupta, D. K. Avasthi and D. Kanjilal, Radiat. Meas., 2004, 38, 197-203.

13 T. Tsvetkova and S. Balabanov, in Ion Beam Applications in Surface and Bulk Modification of Insulators, IAEA, 2008.

14 M. Kawashita, S. Itoh, R. Araki, K. Miyamoto and G. H. Takaoka, J. Biomed. Mater. Res., Part A, 2007, 82A, 995-1003.

15 C. P. Stephens, R. S. Benson, X. Ling, H. Song, H.-J. Ham, R. A. Buchanan and M. Chipara, e-Polym., 2008, 42, 1-11.

16 S. Nouh, A. Abdel Naby and P. J. Sellin, Modification induced by proton irradiation in Makrofol-DE polycarbonate, 2007.

17 E. Ferain and R. Legras, Nucl. Instrum. Methods Phys. Res., Sect. B, 1993, 82, 539-548.

18 E. H. Lee, Nucl. Instrum. Methods Phys. Res., Sect. B, 1999, 151, 29-41.

19 E. Scifoni, E. Surdutovich and A. V. Solov'yov, AIP Conf. Proc., 2009, 1197, 217-227.

20 G. Wang, H. Yi, Y. Li, Y. Wang, D. Liu, F. Gao, W. Liu, J. Ren, X. Wang, Y. Zhao and Y. Wang, Matter and Radiation at Extremes, 2018, 3, 67-77.

21 O. Puglisi, M. Chipara, W. Enge, G. Compagnini, J. ReyesRomero, U. Bacmeister and M. D. Chipara, Nucl. Instrum. Methods Phys. Res., Sect. B, 2000, 166-167, 944-948.

22 A. Tayel, M. F. Zaki, A. B. El Basaty and T. M. Hegazy, J. Adv. Res., 2015, 6, 219-224.

23 L. Calcagno, G. Compagnini and G. Foti, Nucl. Instrum. Methods Phys. Res., Sect. B, 1992, 65, 413-422.

24 H. J. Oh and Y. S. Song, RSC Adv., 2017, 7, 14302-14308.

25 H. J. Oh and Y. S. Song, RSC Adv., 2015, 5, 99797-99805. 